\title{
Effet de la salinité sur la germination graines et la croissance des semis de treize cultivars africains de sésame (Sesamum indicum L.)
}

\section{Adiouma Dangue,}

Laboratoire de Biotechnologies des Champignons, Département de Biologie Végétale, Faculté des Sciences et Techniques, Université Cheikh Anta Diop, Dakar-Fann, Sénégal

\section{Ndiogou Gueye,}

Laboratoire de Biotechnologies des Champignons, Département de Biologie Végétale, Faculté des Sciences et Techniques, Université Cheikh Anta Diop, Dakar-Fann, Sénégal, Institut Sénégalais de Recherche Agricole, Centre pour le Développement de l'Horticulture., Dakar, Sénégal

\section{Ahmed Tidjane Diallo,}

Laboratoire de Biotechnologies des Champignons, Département de Biologie Végétale, Faculté des Sciences et Techniques, Université Cheikh Anta Diop,

Dakar-Fann, Sénégal, Département de Productions Végétales, Ecole

Nationale Supérieure d'Agriculture (ENSA), Université de Thiès, Thiès, Sénégal

Idy Carras Sare, Mame Arama Fall-Ndiaye,

Tahir Abdoulaye Diop,

Laboratoire de Biotechnologies des Champignons, Département de Biologie Végétale, Faculté des Sciences et Techniques, Université Cheikh Anta Diop, Dakar-Fann, Sénégal

Doi:10.19044/esj.2020.v16n15p200 URL:http://dx.doi.org/10.19044/esj.2020.v16n15p200

\section{Résumé}

Le sésame (Sesamum indicum L.), une plante annuelle autoféconde originaire d'Afrique et d'Inde, est très sensible aux stades de germination et de semis mais modérément tolérant aux stress hydrique et salin. Cette étude menée en serre a pour objectif d'évaluer l'effet de différentes concentrations de $\mathrm{NaCl}$ sur la germination des graines et la croissance des semis de treize variétés africaines de sésame provenant du Sénégal, du Burkina Faso, du Cameroun et du Soudan. Un dispositif à blocs randomisés avec deux facteurs et trois répétitions a été utilisé. Les résultats montrent un effet négatif du gradient de salinité sur le taux de germination, le taux de survie, la biomasse 
fraiche, la biomasse sèche, le diamètre au collet et la longueur de la tige. Les variétés AS09, AS13, AS14, AS15, AS19 et AS25 ont été moins affectées par la salinité. L'importance de l'effet dépressif dépend de la concentration de sel et de la variété de sésame.

Mots clés: Sésame, variétés, $\mathrm{NaCl}$, germination, croissance.

\section{Effect Of Salinity on the Germination and Growth of Thirteen African Sesame Cultivars (Sesamum indicum L.)}

\section{Adiouma Dangue,}

Laboratoire de Biotechnologies des Champignons, Département de Biologie Végétale, Faculté des Sciences et Techniques, Université Cheikh Anta Diop,

Dakar-Fann, Sénégal

\section{Ndiogou Gueye,}

Laboratoire de Biotechnologies des Champignons, Département de Biologie Végétale, Faculté des Sciences et Techniques, Université Cheikh Anta Diop, Dakar-Fann, Sénégal, Institut Sénégalais de Recherche Agricole, Centre pour le Développement de l'Horticulture., Dakar, Sénégal

\section{Ahmed Tidjane Diallo,}

Laboratoire de Biotechnologies des Champignons, Département de Biologie Végétale, Faculté des Sciences et Techniques, Université Cheikh Anta Diop,

Dakar-Fann, Sénégal, Département de Productions Végétales, Ecole

Nationale Supérieure d'Agriculture (ENSA), Université de Thiès, Thiès, Sénégal

Idy Carras Sare, Mame Arama Fall-Ndiaye,

Tahir Abdoulaye Diop,

Laboratoire de Biotechnologies des Champignons, Département de Biologie Végétale, Faculté des Sciences et Techniques, Université Cheikh Anta Diop, Dakar-Fann, Sénégal

\section{Abstract}

Sesame (Sesamum indicum L.), an annual self-pollinating plant native to Africa and India, is very sensitive to the stages of germination and sowing but moderately tolerant to water and salt stress. The objective of this 
greenhouse study is to assess the effect of different $\mathrm{NaCl}$ concentrations on seed germination and seedling growth of thirteen African sesame varieties from Senegal, Burkina Faso, Cameroon and Sudan. A randomized block device with two factors and three replicates was used. The results show a negative effect of the salinity gradient on the germination rate, survival rate, fresh biomass, dry biomass, collar diameter and length of the stem. Varieties AS09, AS13, AS14, AS15, AS19 and AS25 were less affected by salinity. The magnitude of the depressive effect depends on the concentration of salt and the sesame variety.

Keywords: Sesame, variety, $\mathrm{NaCl}$, germination, growth.

\section{Introduction}

Le sésame (Sesamum indicum L.) est une oléagineuse annuelle autofécondée, à croissance généralement indéterminée et au port érecté. Cette oléagineuse appartient à la famille des Pedaliaceae et est importante en raison de la résistance de son huile à l'oxydation et à la rancidité. Originaire d'Afrique et d'Inde, elle est cultivée pour ses graines, qui sont riches en huile, en vitamines et en protéines (Islam et al., 2016 ; Mishra et al., 2016 ; Walter et al., 2016). Cependant, sa productivité est limitée par la sécheresse et la salinité à l'instar des autres cultures confrontées aux effets des stress biotiques et abiotiques (Diatta et al., 2014 ; Islam et al., 2016). Certains végétaux disposent d'un potentiel génétique, qui leur confère une tolérance aux stress environnementaux (Diallo et al., 2014). Le sésame est sensible aux stades de semis et de germination, mais modérément tolérant à la sécheresse et à la salinité (Harfi et al., 2016).

La salinisation des sols est répandue dans les zones arides et semiarides. Elle est liée aux conditions climatiques et aux activités humaines. La superficie de terres agricoles touchées par la salinité $(\mathrm{NaCl})$ ne cesse d'augmenter.

Les sols sont dits salés lorsque la CEe (conductivité...) est de 4 dS / m ou plus, ce qui équivaut à environ $40 \mathrm{mM}$ de $\mathrm{NaCl}$ et génère une pression osmotique d'environ 0,2 MPa (Munns \& Tester, 2008 ; Fahramand et al., 2014). La salinité due au $\mathrm{NaCl}$ constitue l'un des principaux stress abiotiques, qui inhibe la croissance des plantes et réduits leur productivité (Fahramand et al., 2014 ; Xie et al., 2016). Au Sénégal, la superficie des terres salées est en permanente augmentation, ce qui a pour corrolaire la baisse de production agricole (Ndiaye et al., 2014). L'amélioration de la production agricole dans les zones affectées par la salinité nécessite la sélection de variétés et de génotypes tolérants aux sels (Benidire et al., 2015).

L'objectif de ce travail est d'évaluer l'effet de différentes concentrations de $\mathrm{NaCl}$ sur la germination des graines et la croissance des semis de treize 
variétés africaines de sésame. Plus spécifiquement, il s'agira de calculer le pourcentage de germination des graines, le taux de survie semis et de determiner les biomasses faiche et sèche, la croisance apicale et le diamètre au collet des plants de sésame.

\section{Materiel Et Methodes}

\section{Matériel végétal et substrat de culture}

Le matériel végétal est constitué des graines de treize variétés africaines de sésame provenant du Sénégal (AD01, AD09, AD14), du Mali (AS09, AS13, AS19), du Bénin (AS23, AS35), du Togo (AS25, AS31), du Burkina Faso (AS22), du Cameroun (AS14) et du Soudan (AS15).

Le substrat est le sol du jardin botanique de la faculté des Sciences et Techniques de l'université Cheikh Anta Diop de Dakar. Des caractéristiques physicochimiques du subtrat sont résumées dans le tableau 1.

Tableau 1: Caractéristiques du sol (substrat) utilisé

\begin{tabular}{|c|c|c|c|c|c|c|c|c|c|c|c|c|c|c|c|c|c|c|c|c|}
\hline $\begin{array}{c}\text { pHeau } \\
1 / 2,5\end{array}$ & $\begin{array}{c}\mathrm{CE} \\
1 / 10 \\
\mu \mathrm{s} / \mathrm{Cm}\end{array}$ & $\% c$ & \%MO & $\% \mathrm{~N}$ & $C / N$ & $\begin{array}{c}\mathrm{Ca} \\
\mathrm{meq} / 100 \mathrm{~g}\end{array}$ & $\begin{array}{c}\mathrm{Mg} \\
\mathrm{meq} / 100 \mathrm{~g}\end{array}$ & $\begin{array}{c}\mathrm{Na} \\
\mathrm{meq} / 100 \mathrm{~g}\end{array}$ & $\begin{array}{c}\mathrm{K} \\
\mathrm{meq} / 100 \mathrm{~g}\end{array}$ & $\begin{array}{c}\mathrm{P} \\
\mathrm{ppm}\end{array}$ & $\begin{array}{c}S \\
\mathrm{meq} / 100 \mathrm{~g}\end{array}$ & $\begin{array}{c}\text { CEC } \\
\text { meq/100g }\end{array}$ & T\% & $\begin{array}{c}\text { PSE } \\
\%\end{array}$ & A\% & LF \% & LG\% & SF \% & SM \% & SG \% \\
\hline 7,4 & 65 & 2,37 & 4,086 & 0,21 & 11 & 6,9 & 0,525 & 0,0425 & 0,139 & 48 & 7,606 & 15 & 51 & 0,3 & 10,75 & 2,5 & 1,29 & 48,315 & 36,66 & 0,485 \\
\hline
\end{tabular}

$\mathrm{T}$ : taux de saturation en bases ; PSE : Pourcentage de Sodium Echangeable ; A : argile ; LF : limon fin ; LG : limon grossier; SF : sable fin ; SM : sable moyen et SG : sable grossier

\section{Dispositif expérimental}

Le dispositif expérimental est constitué de blocs randomisés avec deux facteurs et trois répétitions. Le facteur variété de sésame est constitué de treize modalités (AD01, AD09, AD14, AS09, AS13, AS19, AS23, AS35, AS25, AS31, AS15, AS22, AS14) et le facteur contrainte abiotique $(\mathrm{NaCl})$ comprend cinq modalités $(0 \mathrm{mM} ; 17 \mathrm{mM} ; 34 \mathrm{mM} ; 68 \mathrm{mM} ; 136 \mathrm{mM})$. L'unité expérimentale était un pot contenant $150 \mathrm{~g}$ du substrat de culture. Dans chaque pot, cinq graines d'une varitété ont été semées. L'arrosage à la capacité au champ (Ly et al. 2014) est fait quotidiennement avec la solution correspondante (non saline pour le témoin et saline pour les autres traitements) afin d'éviter tout déficit hydrique.

\section{Paramètres mesurés}

Le nombre de graines germées en terme de levées a été compté chaque jour par pot jusqu'au $21^{\text {ème jour. }}$

Le taux final de germination (TG) et le taux de survie (Ts) sont calculés.

$$
\begin{gathered}
\text { Taux de germination }=\frac{\text { Nombre de graine germées }}{\text { Nombre de graine semées }} \times 100 \\
\text { Taux de survie }=\frac{\text { Nombre de plants vivants }}{\text { Nombre de graine germées }} \times 100
\end{gathered}
$$


Le meilleur plant (le plus développé) de chaque pot est sélectionné pour déterminer les paramètres suivants : diamètre au collet (DC), longueur tige (LT), biomasse fraiche (BF) et biomasse sèche (BS).

Le diamètre au collet est déterminé avec un pied à coulisse à affichage électronique (Ly et al., 2014). La longueur de la tige est mesurée à l'aide d'un double décimètre. La biomasse fraiche est mesurée aussitôt à la récolte et la biomasse sèche après séchage à l'étuve à $70{ }^{\circ} \mathrm{C}$ pendant 48 heures (Ly et al., 2014 ; Faye et al., 2015).

\section{Analyses statistiques}

Les analyses statistiques sont faites avec le logiciel R. Le test de normalité de Shapiro-Wilk est fait pour toutes les données. Le traitement statistique des données à distribution normale est effectué en adoptant une approche paramétrique avec analyse de la variance (ANOVA). Le test de Tukey au seuil de probabilité de $5 \%$ est effectué afin de comparer et classer les moyennes des variables mesurées. Les taux de germination et de survie sont aussi soumis au test de Chi carré de Pearson.

\section{Resultats}

\section{Effet Des Concentrations De NaCl Sur La Germination}

Dans les pots témoins, les plus forts taux de germination (100\%) ont été observés sur les variétés AS09, AS13, AS25 et AS15 et présentent une différence significative avec les autres $(p<0,05)$. Les taux de germination ont été moyens pour les autres cultivars. Les taux de germination ont significativement baissé avec l'augmentation des concentrations en $\mathrm{NaCl}$ (Tableau 2).

Tableau 2 : Taux de germination des variétés de sésame 21 jours après semis à différentes concentrations (a : fort taux de germination).

\begin{tabular}{|c|c|c|c|c|c|}
\cline { 2 - 6 } \multicolumn{1}{c|}{} & \multicolumn{5}{c|}{ Concentrations de NaCl (mM) } \\
\hline Variétés & $\mathbf{0}$ & $\mathbf{1 7}$ & $\mathbf{3 4}$ & $\mathbf{6 8}$ & $\mathbf{1 3 6}$ \\
\hline AD01 & $53,33 \mathrm{abcdef}$ & $73,33 \mathrm{abcd}$ & $33,33 \mathrm{bcdef}$ & $13,33 \mathrm{def}$ & $0 \mathrm{f}$ \\
\hline AD09 & $80,00 \mathrm{abc}$ & $66,67 \mathrm{abcde}$ & $66,67 \mathrm{abcde}$ & $20,00 \mathrm{cdef}$ & 6,67 ef \\
\hline AD14 & $40,00 \mathrm{abcdef}$ & $53,33 \mathrm{abcdef}$ & $66,67 \mathrm{abcde}$ & $33,33 \mathrm{bcdef}$ & $20,00 \mathrm{cdef}$ \\
\hline AS09 & $100,00 \mathrm{a}$ & $100,00 \mathrm{a}$ & $100,00 \mathrm{a}$ & $93,33 \mathrm{ab}$ & $20,00 \mathrm{cdef}$ \\
\hline AS13 & $100,00 \mathrm{a}$ & $93,33 \mathrm{ab}$ & $86,67 \mathrm{ab}$ & $86,67 \mathrm{ab}$ & $13,33 \mathrm{def}$ \\
\hline AS19 & $93,33 \mathrm{ab}$ & $100,00 \mathrm{a}$ & $86,67 \mathrm{ab}$ & $100,00 \mathrm{a}$ & 13,33 def \\
\hline AS23 & $60,00 \mathrm{abcdef}$ & $80,00 \mathrm{abc}$ & $80,00 \mathrm{abc}$ & $53,33 \mathrm{abcdef}$ & $0 \mathrm{f}$ \\
\hline AS35 & $93,33 \mathrm{ab}$ & $93,33 \mathrm{ab}$ & $86,67 \mathrm{ab}$ & $53,33 \mathrm{abcdef}$ & $13,33 \mathrm{def}$ \\
\hline $\mathbf{A S 2 5}$ & $100,00 \mathrm{a}$ & $100,00 \mathrm{a}$ & $86,67 \mathrm{ab}$ & $86,67 \mathrm{ab}$ & $20,00 \mathrm{cdef}$ \\
\hline $\mathbf{A S 3 1}$ & $80,00 \mathrm{abc}$ & $86,67 \mathrm{ab}$ & $73,33 \mathrm{abcd}$ & $66,67 \mathrm{cdef}$ & $0 \mathrm{f}$ \\
\hline AS15 & $100,00 \mathrm{a}$ & $100,00 \mathrm{a}$ & $93,33 \mathrm{ab}$ & $86,67 \mathrm{ab}$ & $20,00 \mathrm{cdef}$ \\
\hline AS22 & $66,67 \mathrm{abcde}$ & $66,67 \mathrm{abcde}$ & $93,33 \mathrm{ab}$ & $66,67 \mathrm{abcde}$ & $0 \mathrm{f}$ \\
\hline
\end{tabular}




\begin{tabular}{|c|c|c|c|c|c|}
\hline AS14 & $93,33 \mathrm{ab}$ & $93,33 \mathrm{ab}$ & $100,00 \mathrm{a}$ & $60,00 \mathrm{abcdef}$ & $13,33 \mathrm{def}$ \\
\hline \multicolumn{5}{|c|}{ Test de Tukey au seuil de 0,5\% : CV=27.18585; PS $<2^{\mathrm{e}-16 * * *}$} \\
Pearson's Chi-squared test X-squared $=11205, \mathrm{df}=194, \mathrm{p}$-value $<2.2^{\mathrm{e}-16}$ \\
\hline
\end{tabular}

Signif. codes: 0 ‘***’ $0.001^{\text {'**’ }} 0.01^{\text {'*’ }} 0.05^{\prime}$ '’ $0.1^{\text {' } ’ 1}$

Sur une même colonne les moyennes affectées de lettres différentes permettent de les classe

en différents groupes du plus fort taux (a) au plus faible (f).

\section{Effet des concentrations de $\mathrm{NaCl}$ sur le taux de survie}

En absence de $\mathrm{NaCl}$, les plus forts taux de survie (100\%) ont été observés sur les variétés AS09, AS13, AS25 et AS15. Sur le reste des variétés des taux de survie moyens ont été enregistés. Les taux de survie ont significativement diminué avec la salinité (Tableau 3).

Tableau 3: Taux de survie des variétés de sésame 21 jours après semis à différentes concentrations de $\mathrm{NaCl}$ (a : fort taux de survie).

\begin{tabular}{|c|c|c|c|c|c|}
\hline \multirow[b]{2}{*}{ Variétés } & \multicolumn{5}{|c|}{ Concentrations de $\mathrm{NaCl}(\mathrm{mM})$} \\
\hline & $\mathbf{0}$ & 17 & 34 & 68 & 136 \\
\hline AD01 & 40,00 defg & 66,67 abcd & 20,00 efg & $0 \mathrm{~g}$ & $0 \mathrm{~g}$ \\
\hline AD09 & 73,33 abcd & $60,00 \mathrm{abcde}$ & $66,67 \mathrm{abcd}$ & $0 \mathrm{~g}$ & $0 \mathrm{~g}$ \\
\hline AD14 & 40,00 defg & 53,33 bcde & 53,33 bcde & $0 \mathrm{~g}$ & $0 \mathrm{~g}$ \\
\hline AS09 & $100,00 \mathrm{a}$ & $100,00 \mathrm{a}$ & 73,33abcd & $6,67 \mathrm{fg}$ & $0 \mathrm{~g}$ \\
\hline AS13 & $100,00 \mathrm{a}$ & $93,33 \mathrm{ab}$ & 73,33abcd & $0 \mathrm{~g}$ & $0 \mathrm{~g}$ \\
\hline AS19 & 80,00 abcd & $100,00 \mathrm{a}$ & 73,33abcd & $6,67 \mathrm{fg}$ & $0 \mathrm{~g}$ \\
\hline AS23 & 53,33 bcde & 73,33 abcd & $60,00 \mathrm{abcde}$ & $0 \mathrm{~g}$ & $0 \mathrm{~g}$ \\
\hline AS35 & $86,67 \mathrm{abc}$ & $86,67 \mathrm{abc}$ & $60,00 \mathrm{abcde}$ & $0 \mathrm{~g}$ & $0 \mathrm{~g}$ \\
\hline AS25 & $100,00 \mathrm{a}$ & $93,33 \mathrm{ab}$ & 73,33abcd & $6,67 \mathrm{fg}$ & $0 \mathrm{~g}$ \\
\hline AS31 & $80,00 \mathrm{abcd}$ & $80,00 \mathrm{abcd}$ & $60,00 \mathrm{abcde}$ & $0 \mathrm{~g}$ & $0 \mathrm{~g}$ \\
\hline AS15 & $100,00 \mathrm{a}$ & $100,00 \mathrm{a}$ & 73,33abcd & $6,67 \mathrm{fg}$ & $0 \mathrm{~g}$ \\
\hline AS22 & $66,67 \mathrm{abcd}$ & $46,67 \mathrm{cdef}$ & 53,33 bcde & $0 \mathrm{~g}$ & $0 \mathrm{~g}$ \\
\hline AS14 & $93,33 \mathrm{ab}$ & $86,67 \mathrm{abc}$ & $86,67 \mathrm{abc}$ & $0 \mathrm{~g}$ & $0 \mathrm{~g}$ \\
\hline
\end{tabular}

Sur une même colonne les moyennes affectées de lettres différentes permettent de les classer en différents groupes du plus fort taux (a) au plus faible (g).

\section{Effet des concentrations de $\mathrm{NaCl}$ sur la longueur des tiges}

La figure 1 présente les variations de la longueur des tiges des treize variétés africaines de sésame en fonction des différentes concentrations de $\mathrm{NaCl}$. Les plus longues tiges ont été obtenues avec les traitements témoins $(0$ $\mathrm{mM}$ de $\mathrm{NaCl}$ ). Des tiges de longueurs moyennes ont été observées à $17 \mathrm{mM}$, celles de faibles longueurs ont été enregistrées pour toutes les variétés de sésame à la concentration $34 \mathrm{mM}$ de $\mathrm{NaCl}$. 


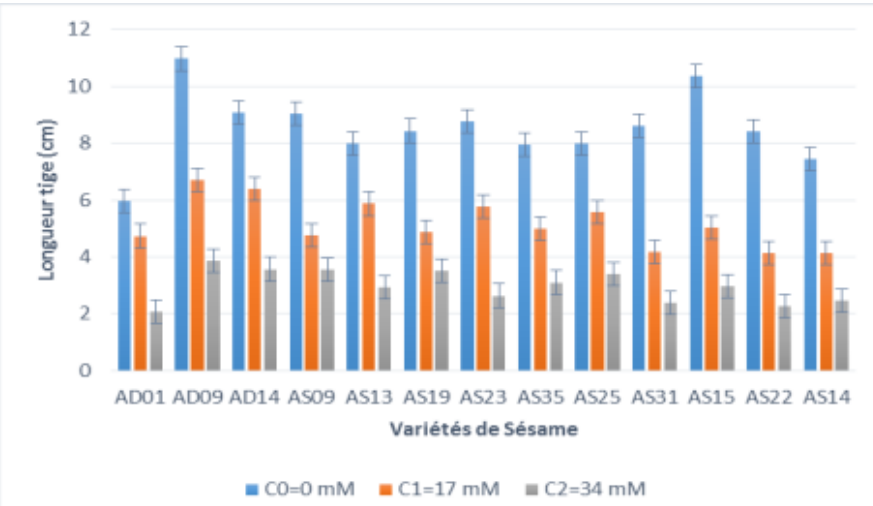

Figure 1 : Longueur de la tige des variétés de sésame à différentes concentrations de $\mathrm{NaCl}$.

\section{Effet des concentrations de $\mathrm{NaCl}$ sur le diamètre au collet}

La figure 2 présente les variations du diamètre au collet des treize variétés africaines de sésame en fonction des différentes concentrations de $\mathrm{NaCl}$. Les plus grands diamètres au collet ont été obtenus au niveau des traitements témoins $(0 \mathrm{mM})$. Des diamètres au collet moyens ont été observés pour toutes les variétés à $17 \mathrm{mM}$ de sel. Les plus petits diamètres au collet ont été enregistrés à $34 \mathrm{mM}$ de $\mathrm{NaCl}$ pour toutes les variétés de sésame

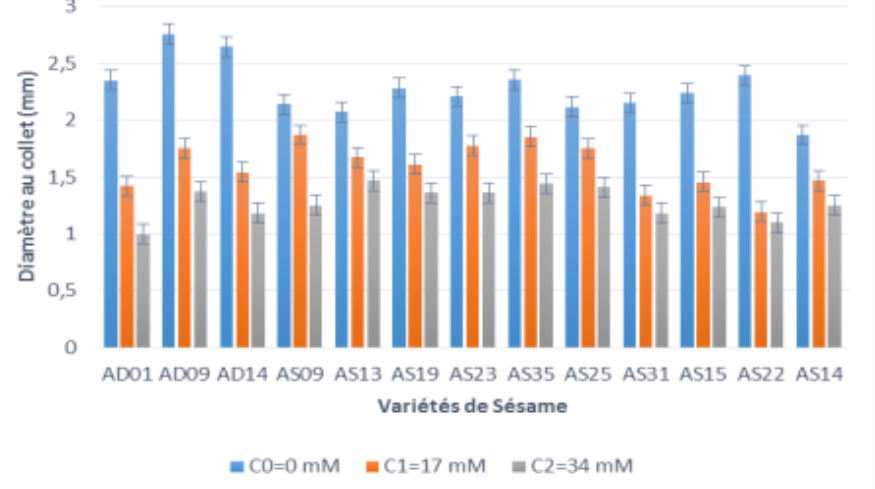

Figure 2 : Diamètre au collet des variétés de sésame en fonction de la concentration de $\mathrm{NaCl}$.

\section{Effet des concentrations de $\mathrm{NaCl}$ sur la biomasse fraiche}

La figure 3 présente les variations de la biomasse fraiche des treize variétés africaines de sésame en fonction des différentes concentrations de $\mathrm{NaCl}$. Les plus importantes biomasses fraiches ont été obtenues pour toutes les variétés en absence de sel. Des biomasses fraiches moyennes ont été observées en présence de $17 \mathrm{mM}$ de $\mathrm{NaCl}$ et de faibles biomasses fraiches ont été enregistrées à $34 \mathrm{mM}$ pour les treize variétés africaines. 


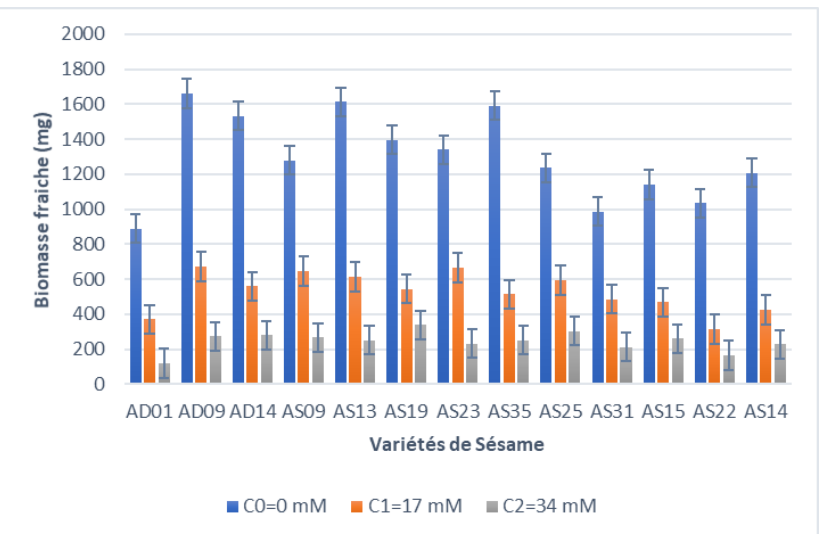

Figure 3: Biomasse fraiche des variétés de sésame en fonction de la concentration de $\mathrm{NaCl}$.

\section{Effet des concentrations de $\mathrm{NaCl}$ sur la biomasse sèche}

La figure 4 présente les variations de la biomasse sèche des treize variétés africaines de sésame en fonction des différentes concentrations de $\mathrm{NaCl}$. Les plus importantes biomasses sèches ont été obtenues à l'absence de $\mathrm{NaCl}$. Des biomasses sèches moyennes ont été observées pour toutes les variétés à $17 \mathrm{mM}$. Les plus faibles biomasses sèches ont été enregistrées à 34 $\mathrm{mM}$ de $\mathrm{NaCl}$ pour toutes les variétés africaines de sésame.

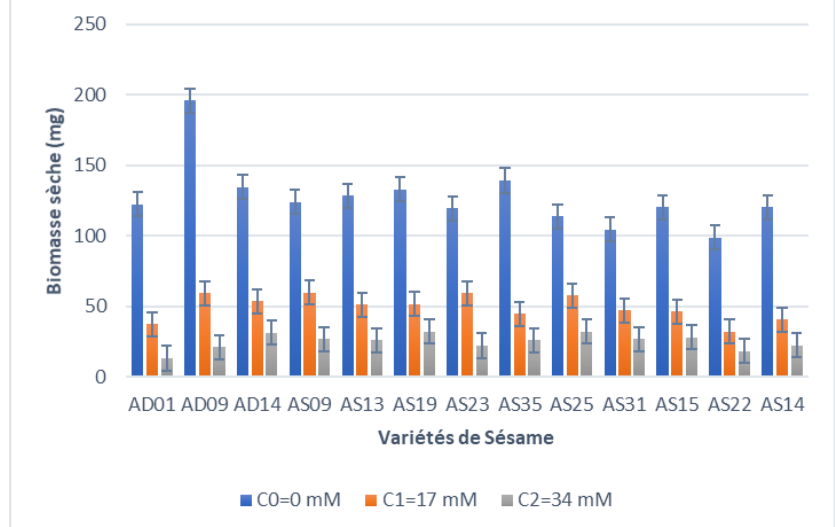

Figure 4: Biomasse sèche des variétés de sésame à différentes concentrations de $\mathrm{NaCl}$.

\section{Discussion}

Les taux de germination les plus importants (100\%) ont été obtenus en absence de salinité avec les variétés de sésame AS09, AS13, AS25 et AS15 (Tableau 2). Des taux de survie de $100 \%$ sont observés pour les témoins des variétés AS09, AS13, AS25 et AS15 (Tableau 3). Le taux de germination a globalement diminué avec l'augmentation de la concentration de sel pour les différentes variétés de sésame (Tableau 2). Ces résultats sont en accord avec ceux de Ndiaye et al. (2014) qui ont montré une diminution du taux de germination de Gossypium hirsutum L. avec l'augmentation de la salinité. 
L'effet dépressif du sel sur la capacité de survie des plants dépend de la concentration en $\mathrm{NaCl}$ dans le milieu de culture et de la variété (Hamrouni et al., 2008 ; Diallo et al., 2014).

Les concentrations de $17 \mathrm{mM}$ et $34 \mathrm{mM}$ de $\mathrm{NaCl}$ ont entrainé la diminution de la longueur de la tige, du diamètre au collet, des biomasses fraiche et sèche (Figures 1 à 4). En effet, une augmentation des concentration en $\mathrm{NaCl}$ inhibe la croissance des plantes (Gehlot et al., 2005).

Les biomasses fraiche et sèche de toutes les variétés sont réduites par la salinité (Figure 3 et 4). Ces résultats rapellent ceux de Bekele et al. (2017), qui ont montré une réduction proportionnelle au niveau de salinité des biomasses fraiche et sèche des plants de différents variétés de sésame.

La salinité a occasionné la diminution du diamètre au collet. Cette même observation a été faite par Bahrami et al. (2016), qui ont montré que la salinité affecte négativement la croissance des plantes. En outre, des observations similaires ont été faites par Ly et al. (2014) sur le diamètre au collet de Jatropha curcas L..

Le taux de germination, la longueur de la tige, la biomasse fraiche et la biomasse sèche ont diminué de façon significative au sein de toutes les variétés de sésame avec l'augmentation des concentrations de $\mathrm{NaCl}$ (Tableau 1 et Figure 2 à 4). Ouji et al. (2015) ont obtenu des résultats similaires en montrant une diminution significative des paramètres de croissance de cinq variétés de lentilles (Lens culinaris L.) avec l'augmentation de la salinité.

\section{Conclusion}

L'effet de la salinité sur la germination et la croissance de treize variétés africaines de sésame a été évalué en serre pendant vingt-un jours. Le taux de germination des graines, le taux de survie, le diamètre au collet, les biomasses fraiche et sèche et la longueur de la tige des plants ont diminué avec l'augmentation de la concentration de $\mathrm{NaCl}$. Les variétés AS09, AS13, AS19, AS25, AS15 et AS14 ont été les plus tolérantes à la salinité qui a moins affecté leurs taux de germination et de survie. Ces résultats révèlent que les variétés de sésame AS09, AS13, AS19, AS25, AS15 et AS14 pourraient être utilisées dans les zones arides et semi-arides où la salinité ne cesse d'augmenter.

Conflits D'interets : Les auteurs déclarent n'avoir aucun conflit d'intérêts.

Contributions Des Auteurs: AD a élaboré et mis au point le protocole expérimental, l'analyse et l'interprétation des données et la rédaction de l'article. NG et ICS sont intervenus dans l'élaboration et la mise au point du protocole expérimental, l'analyse et l'interprétation des données. ATD et MAF-N ont apporté leur contribution dans la correction et à la révision du contenu sur le fond que sur la forme. TAD est le superviseur de ce travail de 
recherche. Il est intervenu dans la correction du protocole, et a coordonné toutes les activités.

\section{References:}

1. Asgharipour, M. R., \& Rafiei, M. (2011). Effect of salinity on germination and seedling growth of lentils. Journal of Applied Sciences Research, 7(11).

2. Bahrami, H., Jafari, A. O., \& Razmjoo, J. (2016). Effect of Salinity Levels $(\mathrm{NaCl})$ on Yield, Yield Components and Quality Content of Sesame (Sesamum indicum L.) Cultivars. Environmental Management and Sustainable Development, 104. https://doi.org/10.5296/emsd.v5i2.9852

3. Bekele, A., Besufekad, Y., Adugna, S., \& Yinur, D. (2017). Screening of selected accessions of Ethiopian sesame (Sesame indicum L.) for salt tolerance. Biocatalysis and Agricultural Biotechnology, 9, 82-94. https://doi.org/10.1016/j.bcab.2016.11.009

4. Benidire, L., Daoui, K., Fatemi, Z. A., Achouak, W., Bouarab, L., \& Oufdou, K. (2015). Effet du stress salin sur la germination et le développement des plantules de Vicia faba L.(Effect of salt stress on germination and seedling of Vicia faba L.). Journal of Materials and Environmental Science, 6(3), 840-851.

5. Diallo, B., Samba, S., Sane, D., \& Diop, T. (2014). Effet du chlorure de sodium sur la germination de graines de \&lt;i\&gt;Ricinus communis\&lt;/i\&gt; L. International Journal of Biological and Chemical Sciences, 7(4), 1534. https://doi.org/10.4314/ijbcs.v7i4.10

6. Djatta, M., Laminou Manzo, O., Macoumba Diouf, P., \& Diop, T. (2014). Effets de l'inoculation mycorhizienne sur le sesame (\&lt;i\&gt;Sesamum indicum\&lt;/i\&gt; L.) en conditions naturelles. International Journal of Biological and Chemical Sciences, 7(5), 2050. https://doi.org/10.4314/ijbcs.v7i5.22

7. Fahramand, M., Mahmoody, M., Keykha, A., Noori, M., \& Rigi, K. (2014). Influence of abiotic stress on proline, photosynthetic enzymes and growth. Int Res J Appl Basic Sci, 8(3), 257-265.

8. Faye, E., Camara, M., Toure, M., \& Mbaye, A. (2015). Evaluation et amélioration du comportement de \&lt;i\&gt;Atriplex lentiformis\&lt;/i\&gt; (Torr.) S. Watson en milieux salés au Sénégal. International Journal of Biological and Chemical Sciences, 8(4), 1697. https://doi.org/10.4314/ijbcs.v8i4.30

9. Gehlot, H. S., Purohit, A., \& Shekhawat, N. S. (2005). Metabolic changes and protein patterns associated with adaptation to salinity in Sesamum indicum cultivars. J. Cell Mol. Biol, 4, 31-39. 
10. Hamrouni, L., Abdallah, F. B., Abdelly, C., \& Ghorbel, A. (2008). La culture in vitro: Un moyen rapide et efficace pour sélectionner des génotypes de vigne tolérant la salinité. Comptes Rendus Biologies, 331(2), 152-163. https://doi.org/10.1016/j.crvi.2007.11.002

11. Harfi, M. E., Hanine, H., Rizki, H., Latrache, H., \& Nabloussi, A. (2016). Effect of Drought and Salt Stresses on Germination and Early Seedling Growth of Different Color-seeds of Sesame (Sesamum indicum). International Journal of Agriculture and Biology, 18(06), 1088-1094. https://doi.org/10.17957/IJAB/15.0145

12. Ibrahim, M. (2016). Response of Sesame (Sesamum indicum L.) to various levels of Nitrogen and Phosphorus in agro-climatic condition of Peshawar. Pure and Applied Biology, 5(1), 121-126. https://doi.org/10.19045/bspab.2016.50016

13. Islam, F., Gill, R. A., Ali, B., Farooq, M. A., Xu, L., Najeeb, U., \& Zhou, W. (2016). Sesame. In Breeding Oilseed Crops for Sustainable Production (p. 135-147). Elsevier. http://linkinghub.elsevier.com/retrieve/pii/B9780128013090000069

14. Ly, M., Kumar, D., Diouf, M., Nautiyal, S., \& Diop, T. (2014). Effet de la salinité sur la croissance et la production de biomasse de deux provenances de \&lt;i\&gt;Jatropha curcas\&lt;/i\&gt; L. cultivés en serre. International Journal of Biological and Chemical Sciences, 8(1), 46. https://doi.org/10.4314/ijbcs.v8i1.5

15. Miraj, S., \& Kiani, S. (2016). Bioactivity of Sesamum indicum : A review study. Der Pharmacia Lettre, 8(6), 328-334.

16. Mishra, P. K., Paroha, S., \& Prakash Mishra, R. (2016). Assessment of Storage Dependent Physiological Parameters of Sesamum indicum seeds. International Journal of Current Microbiology and Applied Sciences, $5(1)$, 641-653. https://doi.org/10.20546/ijcmas.2016.501.065

17. Munns, R., \& Tester, M. (2008). Mechanisms of Salinity Tolerance. Annual Review of Plant Biology, 59(1), 651-681. https://doi.org/10.1146/annurev.arplant.59.032607.092911

18. Ndiaye, A., Faya, E., \& Touré, M. (2014). Effets du stress salin sur la germination des graines de \&lt;i\&gt;Gossypium hirsutum\&lt;/igt; L. Journal of Applied Biosciences, 80(1), 7081. https://doi.org/10.4314/jab.v80i1.5

19. Ouji, A., El-Bok, S., Mouelhi, M., Younes, M. B., \& Kharrat, M. (2015). Effect of salinity stress on germination of five Tunisian lentil (Lens culinaris L.) genotypes. 13 European Scientific Journal vol.11, No.21 ISSN: 1857 - 7881 (Print) e - ISSN 1857- 7431.

20. Walter, O.-A., Patrick, R., Paul, G., \& Patrick, O. (2016). Genotype by environment interaction in sesame (Sesamum indicum L.) cultivars in 
Uganda. African Journal of Plant Science, 10(10), 189-202. https://doi.org/10.5897/AJPS2016.1426

21. Xie, J., Dai, Y., Mu, H., De, Y., Chen, H., Wu, Z., Yu, L., \& Ren, W. (2016). Physiological and biochemical responses to nacl salinity stress in three roegneria (Poaceae) species. Pak. J. Bot, 48(6), 2215-2222. 